\title{
Perfil epidemiológico das internações pelo uso/abuso de drogas na região centro-oeste de Minas Gerais
}

Recebido em: 10/03/2013

Aprovado em: 16/05/2014
Conrado Augusto Ferreira de Oliveira' Geralda de Moraes Teixeira'

Vanessa Pereira Silva ${ }^{1}$

Leandro Santos Ferreira²

Richardson Miranda Machado 3

Resumo: O estudo teve como objetivo caracterizar a hospitalização pelo uso/abuso de drogas. Estudo retrospectivo e exploratório realizado com 6.380 pacientes. Como resultados as características predominantes foram sexo masculino, idade 31-40 anos; hospitalizado pelar própria família e com tempo de permanência superior a 60 dias.O principal financiador das internações foi o sistema de saúde pública com 4.142 (64,4\%) internações pagas. Conclui-se que o uso/abuso de drogas gera um maior grau de dependência e resulta em uma maior dificuldade em alcançar e manter a recuperação Portanto é importante o diagnóstico precoce para evitar hospitalizações e a gravidade dos casos.

Descritores: Usuários de Drogas, Internação, Enfermagem

\section{Epidemiological profile of admissions by the use/abuse of drugs in the central-west region of Minas Gerais}

Abstract:The study aimed to characterize the hospitalization by the use/abuse of drugs. Retrospective and exploratory study conducted of 6380 patients. As a result the predominant features were male, age 31-40 years; hospitalized by family own with and length of stay exceeding 60 days. The main funder of hospitalizations was the health public system with 4142 (64,4\%) paid admissions. Conclusion: Concluded that the use/drug abuse generates a higher level of dependence and results in a greater difficulty in achieving and maintaining recovery Therefore is important the early diagnosis to prevent hospitalizations and severity of cases.

Descriptors: Drug Users, Hospitalization, Nursing

\section{Perfil epidemiológico de los ingresos por el uso y abuso de drogas en la región centro-oeste de Minas Gerais}

Resumen: Caracterizar las hospitalizaciones por el uso/abuso de drogas. Métodos: estudio retrospectivo, exploratorio que incluyó 6380 pacientes. Resultados: las características predominantes fueram hombres, edad 31-40 años, llevados al hospital por la propia familia y la duración de la internación superior a 60 dias. La principal fuente de financiación del hospitalización fue el sistema de salud pública, con 4.142 (64,4\%) ingresos pagados. Conclusión: el uso/abuso de drogas genera un mayor grado de dependencia y se traduce en una mayor dificultad para lograr y mantener la recuperación. Por lo tanto es importante el diagnóstico precoz para prevenir las hospitalizaciones y la gravedad de los casos.

Descriptores: Drogas, Hospitalización, Enfermería

\section{INTRODUÇÃO}

U m dos problemas enfrentados tanto pela sociedade, autoridades, poder público e entidades de saúde, é o crescimento do consumo de drogas psicotrópicas. Esse aumento gera desafios para a saúde pública no Brasil, pelas proporções ainda pouco conhecidas e de grande impacto familiar e social.

Tem-se, assim, de um lado o usuário de substâncias psicotrópicas (e sua família) em situação de vulnerabilidade e fragilidade e, de outro, a necessidade de um tratamento complexo com abordagem multiprofissional e interdisciplinar, em serviços ainda pouco estruturados e experientes no atendimento a esta clientela'.

Segundo a Organização Mundial de Saúde (OMS), droga é qualquer substância não produzida pelo organismo que tem a propriedade de atuar sobre um ou mais de seus sistemas, produzindo alterações em seu funcionamento. Drogas de abuso são definidas como substâncias consumidas por qualquer via de administração, que alteram o humor, nível de percepção ou funcionamento do sistema nervoso central. Dados demonstram que o enfrentamento desta problemática aumenta cada vez mais em proporções mundiais, cerca de $10 \%$ das populações dos centros urbanos de todo o mundo consomem abusivamente substâncias psicoativas, independentemente de idade, sexo, nível de instrução e poder aquisitivo ${ }^{(2,4)}$.

Nessa perspectiva, desenvolvemos este estudo, objetivando caracterizar os pacientes acometidos e, consequentemente, suas internações hospitalares pelo uso/abuso de drogas na região centro-oeste de Minas Gerais, quanto ao sexo, idade, procedência, diagnóstico da internação, tempo de internação na (CSBM), tipo de alta hospitalar, tipo de internação e fonte financiadora da internação.

\section{METODOLOGIA}

Estudo retrospectivo e exploratório, realizado na Clínica São Bento Menni (CSBM), um hospital psiquiátrico filantrópico que presta cuidados no atendimento de urgência, ambulatorial, hospital dia e internação, com capacidade total instalada de 137 leitos. Essa clínica, situada no município de Divinópolis - MG, é considerada centro de referência do Sistema Único de Saúde (SUS) para internações psiquiátricas na região Centro-Oeste de Minas Gerais, atendendo pacientes portadores de transtornos mentais para a propedêutica, tratamento psiquiátrico e ambulatorial. Possui uma clientela diversificada, sendo que a maioria dos seus pacientes (cerca de 85\%) são atendidos pelo SUS, sendo que grande parte é proveniente de cidades circunvizinhas ${ }^{(5)}$.

A pesquisa foi realizada mediante coleta de informações no banco de dados do SIH/CSBM, após aprovação do Comitê de Ética em Pesquisa da Universidade de São Paulo (USP). Com base no que se foi coletado, foi construído um novo banco de dados com informações sobre os pacientes em uso/abuso de drogas, atendendo as seguintes variáveis: sexo, idade, procedência do paciente, diagnóstico da internação, tempo de internação, tipo de

'Enfermeiro Residente em Atenção Básica/Saúde da Família, Universidade Federal de São João Del-Rei. Divinópolis (MG). UFSJ. Email:conradoaugusto@yahoo.com.br ${ }^{2}$ Aluno de Graduação em Enfermagem, Universidade Federal de São João Del-Rei. Divinópolis (MG)-UFSJ.

${ }^{3}$ Enfermeiro, Doutor em Saúde Mental, Universidade de São Paulo. Professor Adjunto do Curso de Graduação em Enfermagem, Universidade Federal de São João Del-Rei. UFSJ. Divinópolis (MG). 
alta hospitalar, tipo de internação e fonte financiadora.

Foram adotados os seguintes critérios de inclusão: 1) pacientes internados na CSBM, no período de 20 de outubro de 1980 (data de inauguração da clinica) a 31 de dezembro de 2005; 2) com permanência na CSBM superior a 24 horas; 3 ) que foram acompanhados desde a admissão, alta ou transferência. Os dados foram analisados pelo Software Statiscal Package for Social Sciences (SPSS) versão 11.5.

\section{RESULTADOS E DISCUSSÃO}

No período estudado dos 24.161 pacientes admitidos na CSBM, 6.380 pacientes foram internados devido ao uso/abuso de drogas, o que representa uma taxa de $26.4 \%$.

Tabela 1 - Caracterização dos pacientes internados na CSBM pelo uso/abuso de drogas.Divinópolis, 2010.

\begin{tabular}{|c|c|}
\hline Variável & Freqüência (n \%) \\
\hline \multicolumn{2}{|l|}{ 1-Sexo } \\
\hline $\begin{array}{l}\text { Masculino } \\
\text { Feminino }\end{array}$ & $\begin{array}{l}4325(67.78 \%) \\
2005(32.22 \%)\end{array}$ \\
\hline \multicolumn{2}{|l|}{2 - Idade } \\
\hline $\begin{array}{l}10 \text { a } 20 \\
21 \text { a } 30 \\
31 \text { a } 40 \\
41 \text { a } 50 \\
51 \text { a } 60 \\
61 \text { a } 70 \\
>70\end{array}$ & $\begin{array}{l}178(2.78 \%) \\
1670(26.17 \%) \\
2133(33.43 \%) \\
1559(24.43 \%) \\
745(11.67 \%) \\
89(1.39 \%) \\
06(0.09 \%)\end{array}$ \\
\hline
\end{tabular}

\section{3 - Procedência}

$\begin{array}{ll}\text { Consultório Médico Particular } & 797(12.49 \%) \\ \text { Centros de Atenção Psicossocial } & 2470(38.71 \%) \\ \text { Família } & 2793(43.77 \%) \\ \text { Outros } & 320(5.01 \%)\end{array}$

\section{4 - Diagnóstico da Internação}

$\begin{array}{ll}\text { F10- Uso de álcool } & 1220(19.12 \%) \\ \text { F11- Uso de opiáceos } & 137(2.14 \%) \\ \text { F12- Uso de canabinóides } & 1150(18.02 \%) \\ \text { F13- Uso de sedativos e hipnóticos } & 22(0.34 \%) \\ \text { F14- Uso da cocaína/crack } & 1380(21.63 \%) \\ \text { F15- Uso de outros estimulantes } & 11(0.17 \%) \\ \text { F16- Uso de alucinógenos } & 07(0.10 \%) \\ \text { F17- Uso de fumo } & 01(0.15 \%) \\ \text { F18- Uso de solventes voláteis } & 17(0.26 \%) \\ \text { F19- Uso de múltiplas drogas } & 2435(38.16 \%)\end{array}$

\section{5 - Tempo de Internação na CSBM}

1 a 30 dias

31 a 60 dias

$997(15.62 \%)$

$1483(23.24 \%)$

61 a 90 dias

$2149(33.68 \%)$

$>90$ dias

$1751(27.44 \%)$

\section{6- Tipo de Alta Hospitalar}

Alta Médica Hospitalar

Alta a Pedido

Alta Administrativa

Alta por Abandono/Evasão

Transferência Clínica

$3.315(51.95 \%)$

928(14.54\%)

$181(2.83 \%)$

$434(6.80 \%)$

$1522(23.85 \%)$

\section{7- Tipo de Internação}

\section{Internações}

Reinternações

$3851(60.36 \%)$

2529 ( $39.63 \%)$

\section{8 - Fonte Financiadora da Internação}

$\begin{array}{ll}\text { Sistema Único de Saúde } & 4142(64.92 \%) \\ \text { Particular } & 877(13.74 \%) \\ \text { Convênios } & 1355(21.23 \%) \\ \text { Gratuito } & 6(0.09 \%)\end{array}$

Fonte: banco de dados do SIH/CSBM
Após a análise dos dados verificou-se a predominância do sexo masculino com 4.325 (69.7\%) sob o sexo feminino com 1880 (30.2\%) pacientes. Estudos sobre o uso/abuso de drogas também identificaram o sexo masculino como o maior consumidor, fato este relacionado a questões histórico-culturais explicadas pelo fato de que o sexo feminino procura menos os serviços de saúde por questões de gênero, estigma social, autoimagem, o papel feminino da mulher perante a sociedade e por conseguir esconder a dependência, principalmente em relação ao álcool, por mais tempo ${ }^{(6-7)}$.

Com relação a faixa etária, observou-se um maior número 2.133 (33.43\%) de internações na faixa etária de 31 a 40 anos, seguida da faixa de 21 a 30 anos (26.17\% ). Estudos 8-16 demonstram que a idade usual da busca de tratamento para dependência química se dá por volta dos quarenta anos, que é quando o indivíduo apresenta graves problemas de saúde decorrentes do uso abusivo de álcool e outras drogas consumidas durante um longo período de tempo e exposição a tais substancias fato este que pode estar relacionado ao inicio precoce do envolvimento com drogas, que segundo alguns autores ocorre na adolescência(9).

Quanto a procedência, 2.793 (43.77\%) dos pacientes foram provenientes para internação da própria família e 2.470 (38.71\%) foram advindos do centro de atenção pscicosocial. Pesquisas10,11 demonstram que a família exerce um papel muito importante na recuperação e prevenção da recaída, junto com os profissionais ela facilita a aceitação da internação pelo paciente e minimiza os conflitos advindos desta.

Em relação ao diagnóstico de internação prevaleceu o uso de múltiplas drogas com um total de 2435 (38.16\%), seguido dos diagnostico de uso de cocaína e crack 1380 (21.63\%) e álcool 1220 (19,12\%). Alguns estudos relatam que dentre indivíduos que buscam tratamento para uso de cocaína existe em geral, grande contingente de dependentes ou abusadores de álcool. A combinação entre cocaína e álcool tende a ser feita para reforçar os efeitos potentes da cocaína, quanto ao uso combinado de crack o álcool tem seu uso posterior na tentativa de melhorar a secura da boca, amenizar a intensidade do crack e/ou efeitos indesejáveis. Além disso, o emprego do álcool nesse estágio final tende a diminuir as dosagens de crack utilizadas ${ }^{(13)}$.

Além disso, o uso de múltiplas drogas pode estar relacionado a indivíduos com exposição de longa data ao uso/abuso de substancias, que devido ao período prolongado ocorre o aumento da quantidade e uso de outras substâncias para conseguir o mesmo efeito. Fato este que pode ser observado ao verificarmos que os pacientes dependentes, fazem uso concomitante de outras substâncias psicoativas, destacando-se o tabaco, a maconha e o álcool. Ao verificarmos a cocaína e o crack como segundo grupo de pacientes internados pelo uso, vários autores ${ }^{(14-15)}$, relatam que este fenômeno pode estar relacionado a facilidade de acesso, popularização desta droga, menor risco de contaminação por HIV, o que ocorre entre usuários de drogas injetáveis, o baixo custo do crack e o forte efeito e poder aditivo desta forma desta droga. $\mathrm{O}$ álcool foi a terceira causa de internação o que difere dos estudos (6-16-17), nos quais o álcool foi o principal motivo de internação e a principal substância psicoativa utilizada pelos pacientes dependentes químicos.

Quanto ao tempo de internação, dos 6380 pacientes internados pelo uso/abuso de drogas, 2.149 (33.68\%) tiveram internado entre 61 a 90 dias, 1751 (27.44\%) acima de 90 dias e 1.483(23.24\%) entre 31 a 60 dias. Ao considerarmos que a autorização de internação hospitalar (AIH) emitida pelo SUS é válida para um prazo máximo 
de 30 dias, e que após este tempo pode ser renovada por mais duas vezes, constatamos que a maioria dos pacientes teve um longo período de permanência hospitalar, este fato torna-se mais um elemento que contrapõe o preconizado pela reforma psiquiátrica, a qual preconiza, sobretudo os tratamentos realizados em serviços extra hospitalares e de curta permanência. Estudo11 cujo objetivo foi avaliar a estratégia de reabilitação psicossocial no campo da atenção aos transtornos decorrentes do consumo de álcool e outras drogas demonstraram que não há avaliação da padronização dos serviços psiquiátricos (freqüência, intensidade, concentração de serviços) durante o tratamento da dependência química, principalmente quando associadas a transtornos mentais.

No que se refere a alta hospitalar, 3.315(51.95\%) dos pacientes tiveram alta médica, o que pode ser considerado um bom indicador de adesão ao tratamento. Estudo 16 aponta passos importantes para obter sucesso no tratamento, o primeiro passo é o senso crítico do paciente quanto a dependência química e o reconhecimento deste para necessidade do tratamento. O segundo passo é o tratamento medicamentoso e o terceiro envolve o tratamento psíquico, por fim, o último passo a reinserção social; não adianta o dependente químico estar abstinente e continuar a frequentar os mesmos locais que predispõem e facilitam o consumo, fazendo com ceda a pressão social.

Dos 6380 pacientes internados na CSBM, 3851( 60.36\%) foram internados pela primeira vez em contrapartida os demais pacientes 2529 ( 39.63\%) Algumas condições justificam as internações, como necessidade de tratamento clínico em virtude de complicações associadas à síndrome de abstinência ao álcool, risco de complicações pelo uso/abuso o que proporciona um critério da gravidade da dependência que quando detectadas no início, podem ser tratadas, promovendo a recuperação completa; e alguns a necessidade de tratamento psiquiátrico associado. Acredita-se que para que haja redução das reinternações seja necessário a disponibilidade de serviços extramurais (ambulatórios, hospitais-dia, oficinas protegidas, etc.) que realizam de forma eficiente a farmacoterapia, a psicoterapia, a orientação, a terapia ocupacional e a reabilitação social conforme preconizado.

A maior fonte financiadora foi o Sistema Único de Saúde (SUS), responsável pela internação de 4.142(64.92\%) dos pacientes, o que era de esperar esta prevalência, uma vez que a CSBM tem caráter filantrópico maioria dos leitos SUS, o que contraria a Lei no. 10.216 de 06/04/2001 que dispõe sobre a extinção progressiva dos manicômios e sua substituição por novas modalidades de atendimento, tais como: hospitais-dia, Centros de Atenção Psicossocial - CAPS e Núcleos de Atenção Psicossocial - NAPS 17.

Os convênios foram responsáveis por 1.355 (21.23\%) das internações esse fato pode estar relacionado com atual momento vivido no país da ascensão das classes sociais menos favorecidas decorrentes da melhora do poder aquisitivo. Porém, evidencia que o modelo hospitalcentrico continua personificado como a única referencia para tratamento, necessitando assim da divulgação e conhecimento por parte da população.

\section{CONCLUSÃO}

A predominância do sexo masculino, com idade entre 31 e 40 anos entre os pacientes caracterizados neste perfil epidemiológico, é um dado que requer atenção especial uma vez que se constitui uma importante faixa etária, por ser uma força atuante no mercado de trabalho, e é justamente nessa idade que ocorre a manifestação de sintomas crônicos decorrentes do uso/abuso de drogas. Ressaltamos assim, a necessidade da implantação de abordagens diferenciadas e mais precoces, que ajudem o usuário a ser tratado antes de ter a vida produtiva prejudicada pela dependência.

Por fim, a constatação de que os principais motivos para internação e de diagnostico foram o uso múltiplo de drogas, álcool e crack, percebemos a necessidade de haver maior intervenção por parte das políticas públicas na dependência química. A prevenção voltada para o uso abusivo e/ou dependência de álcool e outras drogas pode ser definida como processo de planejamento, organização e implementação de múltiplas estratégias com a integração de vários sujeitos da sociedade. 


\section{Referências}

1.Rodrigues DS, Backes DS, Freitas HMB, Zamberlan C, Gelhen MH, Colomé JS. Conhecimentos produzidos acerca do crack: uma incursão nas dissertações e teses brasileiras. Ciênc. saúde coletiva [serial on the Internet]. 2012 May [cited 2012 OUT 10] ; 17(5): 1247-1258.

2.Ministério da Saúde (BR) - A política do Ministério da Saúde de atenção integral a usuários de álcool e outras drogas, 2a edição. Brasília: 2004.

3. Carlini EA, Nappo AS, Gualduróz, JCF, Noto AR. Drogas psicotrópicas: o que são e como agem. IMESC: 2001.

4. Galduróz JCF, Noto AR, Fonseca AM, Carlini EA. (Eds.V Levantamento Nacional sobre Consumo de Drogas Psicotrópicas entre Estudantes do Ensino Fundamental e Médio da Rede Pública de Ensino nas 27 Capitais Brasileiras - 2004. Cebrid: São Paulo: 2005.

5. Carlini EA, Galduróz JCF, Noto AR, Nappo SA. II Levantamento domiciliar de drogas psicotrópicas no Brasil: estudo envolvendo as 108 maiores cidades do país 2005. Cebrid/Unifesp, São Paulo, 2005.

6. Clinica Psiquiátrica São Bento Menni: apresentação. [Citado 2012 mar.]. Disponível em <http:// http://www. hospitaleirasbrasil.org/index.php?irmas=Nav/Clinica $>$

7. Peixoto C, Prado CHO, Rodrigues CP,Cheda JND, Mota LBT,Veras AB . Impacto do perfil clínico e sociodemográfico na adesão ao tratamento de pacientes de um Centro de Atenção Psicossocial a Usuários de Álcool e Drogas (CAPSad). J, bras, psiquiatr. 2010: 59 (4) 317-321.

8. Faria JG, Schneider, DR. O perfil dos usuários do CAPSadBlumenau e as políticas públicas em saúde mental. Psicol Soc.2009 Dec. 21 (3): 324-333.

9. Sousa FSP, Oliveira EN. Caracterização das internações de dependentes químicos em Unidade de Internação Psiquiátrica do Hospital Geral. Ciênc. saúde coletiva 2010 May; 15(3): 671-677

10. Mombelli MA, Marcon SS, Costa JB. Caracterização das internações psquiatricas para desintoxicação de adolescentes dependentes químicos: Rev Bras. Enferm. 2010.Out 63(5):735-740.
11. Azevedo DM, Miranda FAN. Praticas profissionais e tratamento ofertado no CAPSad do município de Natal-RN. Esc. Anna Nery.2010. Mar 14 (1) 56-63.

12. Pinho $\mathrm{PH}$, Oliveira $\mathrm{MA}$, Almeida MM. A reabilitação psicossocial na atenção aos transtornos associados ao consumo de álcool e outras drogas: uma estratégia possível?. Rev. psiquiatr. clín. 2008 Mai.35 ( suppl. 1): 82-88.

13. Ministério da saúde (BR).Saúde mental no SUS: as novas fronteiras da reforma Psiquiátrica. Relatório de gestão 20072010. Brasília. 2011.

14. Dias AD, Araújo MR, Laranjeira R. Evolução do consumo de crack em coorte com histórico de tratamento. Rev. Saúde Pública 2011 Out; 45(5): 938-948.

15. Vargens RW, Cruz MS, Santos MA. Comparação entre usuários de crack e de outras drogas em serviço ambulatorial especializado de hospital universitário. Rev. Latino-Am. Enfermagem .2011 June 19(spe): 804-812.

16. Formiga LT, Santos RCS, DumckeTS, Araújo RB.Comparação do perfil de dependentes químicos internados em uma unidade de dependência química de Porto Alegre/RS em 2002 e 2006. Rev HCPA 2009;29(2):120-126.

17. Machado RM. Transtornos Psiquiátricos: Uma abordagem epidemiológica do alcoolismo na região Centro-Oeste de Minas Gerais. Divinópolis. Tese [Doutorado em Enfermagem Psiquiátrica ] - Faculdade de Enfermagem da USP/ Ribeirão Preto; 2010.

18. Donatti G, Mangeon EPE, Andrade AG. Caracterização do gasto SUS com internações de dependentes de substâncias psicoativas no período de 2000 a 2002 no município de Campinas - SP. Rev. psiquiatr. clín.2008.v.35 supl.1:2-7.

19. Brasil. Lei 10.708, de 31 de julho de 2003. Dispõe sobre a regulamentação do auxílio-reabilitação psicossocial, para assistência, acompanhamento e integração social, fora da unidade hospitalar de pessoas acometidas por transtornos mentais, com história de longa internação psiquiátrica (com dois anos ou mais de internação).. Diário Oficial da União de 01 Agosto de 2003. 